\title{
Optimizing Soft Information in Relay Networks
}

\author{
Krishna Srikanth Gomadam, Syed Ali Jafar \\ Center for Pervasive Communications and Computing \\ Electrical Engineering and Computer Science \\ University of California, Irvine, CA 92697-2625 \\ Email:kgomadam@uci.edu, syed@uci.edu
}

\begin{abstract}
We classify relay forwarding schemes on the basis of soft and hard information. While soft information is highly beneficial, it may also incur additional expenditure of network resources such as power and bandwidth. The relay functionality optimization problem is therefore related to optimizing soft information. With SNR as a metric, we show that the optimal soft information relay mechanism for uncoded communication is the estimate and forward (EF) policy. We demonstrate that EF is well suited to both parallel and serial relay networks due to its optimized soft information content. We also provide an example of a hybrid network where the performance gain from $\mathrm{EF}$ is significant.
\end{abstract}

\section{INTRODUCTION}

Network coding that promises enormous performance improvement over routing based networks, allows packets to mix across the network and arrive at the destination through a number of routes [1]. In wireless relay networks with multiple relays, simple schemes such as amplify and forward outperform sophisticated schemes like decode and forward [2]. The performance advantage is due to soft information relaying. If the intermediate nodes instead of decoding the message try to provide partial or soft information about the source transmitted message, the destination can exploit route diversity inherent in the network.

The concept of hard and soft information is illustrated through the following example. Consider an uncoded system with the source employing BPSK modulation. Any collaborating node in the network will receive a noisy version of the transmitted signal.

$$
r=x+n_{1}
$$

The collaborating node has to forward a symbol based on the received symbol subject to its power constraint. There are numerous ways of forwarding the received signal to the final destination; the most common strategies are amplify and forward (AF) and demodulate and forward (DF). An AF relay simply forwards a scaled version of the received signal $r$ where the scaling factor satisfies its power constraint. The AF relay function can be written as

$$
f_{A F}(r)=\sqrt{\frac{P_{R}}{P+1}} r
$$

where $P$ and $P_{R}$ are the source and relay power respectively. In DF schemes, demodulation of the received symbol at the relay is followed by modulation with its own power constraint

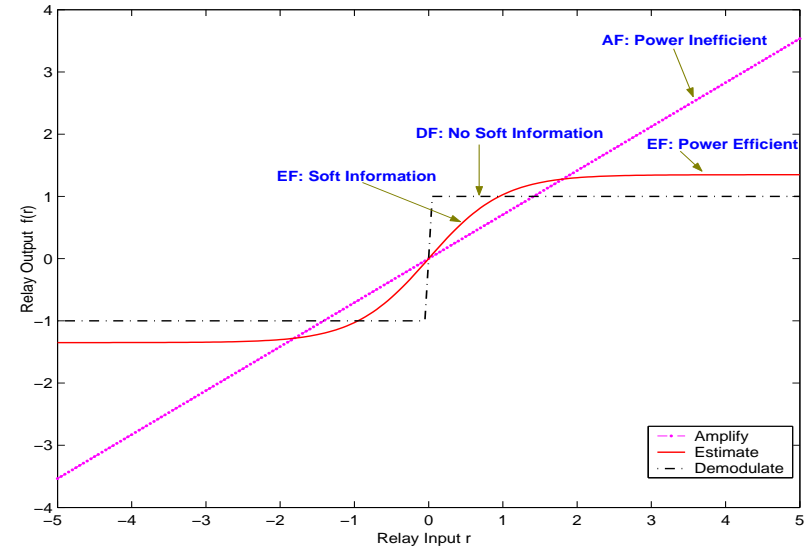

Fig. 1. Relay functions of common forwarding schemes $(P=1)$.

$P_{R}$. The DF relay function for BPSK modulation is given by,

$$
f_{D F}(r)=\sqrt{P_{R}} \operatorname{sgn}(r),
$$

where $\operatorname{sgn}(r)$ outputs the sign of $r$. The relay functions are shown in Fig. 1. It can be seen that the magnitude of the received symbol remains irrelevant during the demodulation process although it contains reliability or soft information. Due to demodulation, the relay transmitted signal carries no information about the degree of uncertainty in the relay's choice of the optimal demodulated symbol. Demodulation at the relays can lead to severe performance degradation in some scenarios. For example, consider a three-relay parallel network with the received symbol at the relays being [0.001 $0.001-1]$. Demodulation at the relays will result in transmission of $\left[\begin{array}{lll}1 & 1 & -1\end{array}\right]$ for $P_{R}=1$. As all the relay transmissions have equal weights, it clearly results in a suboptimal performance. This explains the reason behind DF not achieving full diversity [3]. Evidently with AF, the relay tries to provide soft information to the destination. The relay in addition to the hard decision also indicates the reliability of its decision. Here, the sign of the relay transmitted symbol represents the hard decision; the magnitude provides the reliability information. While the reliability information is greatly beneficial in the uncertainty region, it may not be desirable when $|r|$ is high as significant power is expended. Especially since the relay has an overall power constraint, reliability information for a large $|r|$ reduces the effectiveness of the reliability information at low $|r|$.

From the relay functions of $\mathrm{AF}$ and $\mathrm{DF}$, one can argue 


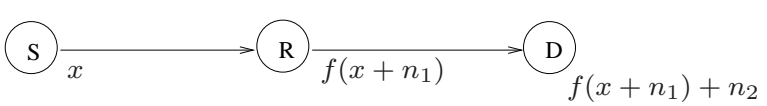

Fig. 2. Elementary Relay Channel

that an optimal relay function should provide soft information when there is an uncertainty in the received symbol. However, at the same time it should conserve power when the cost of power outweighs the value of soft information. The natural question therefore is: when is soft information required and to what degree? In other words, the problem is simply to determine the optimal relay functionality.

The soft information concept also applies for coded transmission. Decode and forward is an example of hard information as the relay tries to hard-decide the source transmitted code. In compress and forward [4], the relay quantizes the received signal using Wyner-Ziv compression, with the level of quantization decided by the channel capacity of the relay destination link. Higher the capacity, the greater is the soft information.

As soft information usually comes at the cost of network resources such as power and bandwidth, it is important to optimize soft information content in the relay forwarding scheme for the network resource constraints. In the next section, we consider the problem of optimizing the relay functionality for a single relay network for the case of uncoded transmission.

\section{SOFT INFORMATION OptimizATION}

Consider an elemental relay channel model as shown in Fig. 2 , in which the relay $\mathrm{R}$ assists the communication between the source $\mathrm{S}$ and the destination D. Both S-R and R-D links are assumed to be non-fading. Without loss of generality, channel gains for the source-relay and the relay-destination link can be incorporated into the model by modifying the source and relay power appropriately. The source and relay power constraints are $P$ and $P_{R}$ respectively. At both the relay and the destination, the received symbol is corrupted by real additive white Gaussian noise of unit power. Relay $\mathrm{R}$ observes $r$, a noisy version of the transmitted symbol $x$. Based on the observation $r$, the relay transmits a symbol $f(r)$ which is received at the destination along with its noise $n_{2}$.

$$
\begin{aligned}
& r=x+n_{1} \\
& y=f(r)+n_{2}
\end{aligned}
$$

The relay function $f$ satisfies the average power constraint, i.e. $\mathcal{E}_{r}\left[|f(r)|^{2}\right]=P_{R}$. We seek to determine the relay function $f($.$) that contains the optimal degree of soft information. We$ employ SNR as an end to end metric to solve the optimization problem. We will later see how SNR is closely related to soft information.

\section{A. Definition of SNR}

The conventional SNR definition cannot be applied directly as the destination received signal $y$ may have an arbitrary nonlinear and probabilistic dependence (contains some soft information about $x$ ) on the desired signal $x$ [5]. Any signal $y$ that contains or correlated with $x$ can be expressed in the form,

$$
y=\frac{\mathcal{E}\left[x^{*} y\right]}{\mathcal{E}\left[|x|^{2}\right]}\left(x+e_{u}\right) .
$$

Rearranging (4),

$$
e_{u}=\frac{P}{\mathcal{E}\left[x^{*} y\right]} y-x .
$$

It is easy to verify that $e_{u}$ is uncorrelated to $x$. The scaling factor $\frac{\mathcal{E}\left[x^{*} y\right]}{\mathcal{E}\left[|x|^{2}\right]}$ in (4) is common to both the signal and error terms. Thus SNR is defined as follows:

$$
\mathrm{SNR}=\frac{\mathcal{E}\left[|x|^{2}\right]}{\mathcal{E}\left[\left|e_{u}\right|^{2}\right]}
$$

The advantage of the generalized definition lies in its applicability to both linear and nonlinear relay functions. The generalized SNR is an indication of what fraction of the signal power contains soft information about the source transmitted signal.

\section{B. Optimal Relay Function}

Theorem 1: For a network with a single relay that has a power constraint $P_{R}$, the relay function that maximizes SNR at the destination is

$$
f(r)=\sqrt{\frac{P_{R}}{\mathcal{E}_{r}\left[|\mathcal{E}(x \mid r)|^{2}\right]}} \mathcal{E}[x \mid r],
$$

regardless of the input and noise distributions.

Proof Outline: First, it can be shown that maximizing SNR at the output of the relay amounts to maximizing SNR at the destination. From the SNR definition in (6), it is clear that SNR maximization is equivalent to minimizing the power of the uncorrelated error associated with the relay function. Interestingly, the estimate that minimizes the mean squared uncorrelated error(MSUE) is a scaled MMSE with a unique scaling factor.

$$
\hat{X}(r)=\frac{P}{\mathcal{E}\left[x^{*} \mathcal{E}[x \mid r]\right]} \mathcal{E}[x \mid r]
$$

As the scaling factor does not affect SNR, any scaled version of MMSE estimator is SNR optimal. For the relay power of $P_{R}$, the SNR maximizing relay function is given by

$$
f(r)=\sqrt{\frac{P_{R}}{\mathcal{E}_{r}\left[|\mathcal{E}(x \mid r)|^{2}\right]}} \mathcal{E}[x \mid r] .
$$

The detailed proof is available in [5].

Fig. 1 shows the the EF relay function for BPSK modulation. It can be seen that the relay function $f_{E F}$ is linear for small values of $|r|$. Its slope reduces gradually and ultimately becomes flat similar to $f_{D F}$. It is intuitively appealing as it eliminates the disadvantages associated with DF and AF. 


\section{Comparative AnAlysis}

For any relay function, SNR at the destination can be obtained from its MSUE. Therefore calculation of MSUE of $\mathrm{DF}$ and $\mathrm{AF}$ allows a direct comparison of these schemes with the SNR optimal EF. To determine the MSUE estimate from any relay function, we only need to obtain the scaling factor that allows the relay function to be expressed as in (4). In this section, we compare the schemes for BPSK modulation.

\section{A. BPSK Modulation}

We express the relay function of a demodulating relay for BPSK as

$$
f_{D F}(x+n)=\sqrt{P_{R}} \operatorname{sign}(x+n)=\sqrt{\frac{P_{R}}{P}}(x+d) .
$$

where $d$ is the Euclidean distance between the input symbol $x$ and the demodulated symbol. The distribution of $d$ conditioned on $x$ is given by

$$
d=\left\{\begin{array}{lr}
0 & 1-\epsilon \\
-2 x & \epsilon
\end{array}\right.
$$

where $\epsilon=Q(\sqrt{P})$, the probability of symbol error. As seen from the error distribution, the demodulation error $d$ is correlated with $x$. The correlation between the input and the error is given by

$$
\mathcal{E}(x d)=-2 P \epsilon .
$$

The uncorrelated error can be calculated from (4). The mean squared uncorrelated error is given by

$$
\operatorname{MSUE}_{D F}=\frac{4 P \epsilon(1-\epsilon)}{(1-2 \epsilon)^{2}} .
$$

At low source transmit power, the mean squared uncorrelated error can be approximated as

$$
\operatorname{MSUE}_{D F}=2 \pi\left(\frac{1}{4}-\frac{P}{2 \pi}\right) .
$$

As $P \rightarrow 0$, the uncorrelated error power shoots up to $\frac{\pi}{2}$. It should be noted that the noise variance at the relay is 1 and hence DF operation increases the error power.

As the relay function of an $\mathrm{AF}$ relay is a scaled version of the received signal $r$, it is simple to determine the MSUE associated with the relay function. From (4), we have

$$
e_{u}=\frac{P}{\mathcal{E}\left[x^{*} f_{A F}(x+n)\right]} f_{A F}(x+n)-x=n .
$$

The MSUE of AF is therefore the same as the noise variance, $\operatorname{MSUE}_{A F}=1$, interestingly independent of the source transmit power. Fig. 3 plots the MSUE as a function of transmit power for all the three schemes. Several interesting observations can be made. It can be seen that $\mathrm{AF}$ is close to optimum (EF) at low $P$ while DF is near optimal at high $P$. In the intermediate range, both $\mathrm{AF}$ and $\mathrm{DF}$ are far from optimal. It is well known that $\mathrm{AF}$ suffers from noise amplification at low SNR [6], which is in contrast to the results here. When we view the relay operation as an estimation, it is only natural that the estimation error is large at low $P$, which results in noise

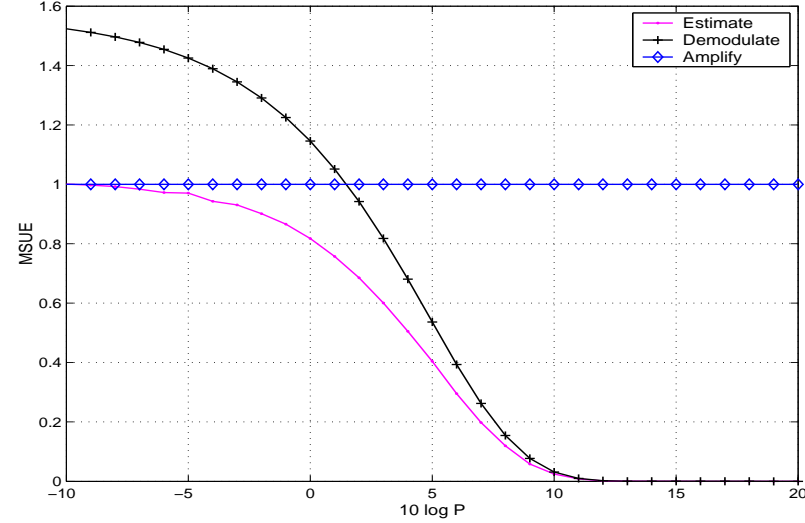

Fig. 3. MSUE vs transmit power for BPSK modulation

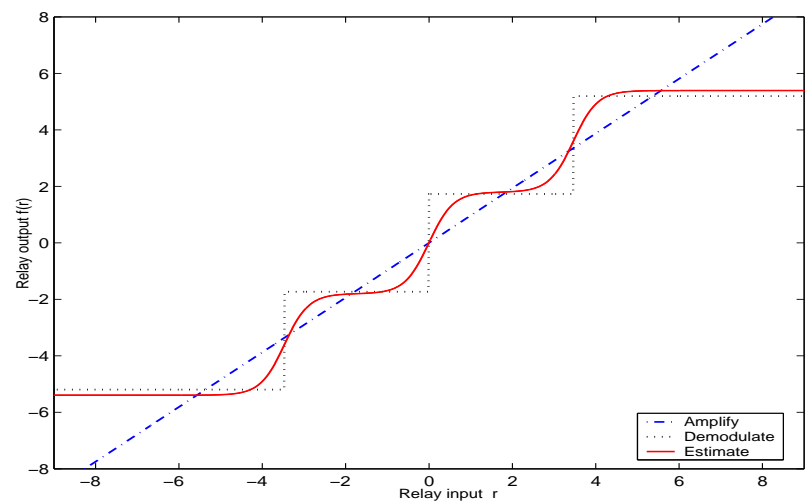

Fig. 4. Relay Functions for 4-PAM modulation

amplification. In fact AF is very close to optimum among all memoryless relay functions at low $P$. Rather it is DF that suffers the most from noise/error amplification. However, AF is inefficient at high $P$ as $\operatorname{MSUE}_{A F}=1$ does not decrease with $P$, while uncorrelated error in $\mathrm{DF}$ and $\mathrm{EF}$ vanishes at high $P$.

\section{B. Higher Order Constellations}

For fixed input power $P$, increasing the number of constellation points $M$ will result in an increased MSUE for EF. As the constellation size increases, the uncertainty about the transmitted symbol increases, thereby reducing the effectiveness of soft information. Fig. 4 shows the relay functions for 4-PAM constellation set. Interestingly, the relay functions of the schemes become more and more similar with increase in constellation points.

For Gaussian inputs, the unconstrained MMSE estimate and the linear MMSE estimate are equivalent.

$$
\mathcal{E}[x \mid r]=\frac{P}{P+1} r
$$

Thus AF and EF strategies are the same for a Gaussian source. In this context, it can also be shown that $\mathrm{DF}$ and $\mathrm{AF}$ are equivalent for Gaussian inputs. The notion of demodulation 


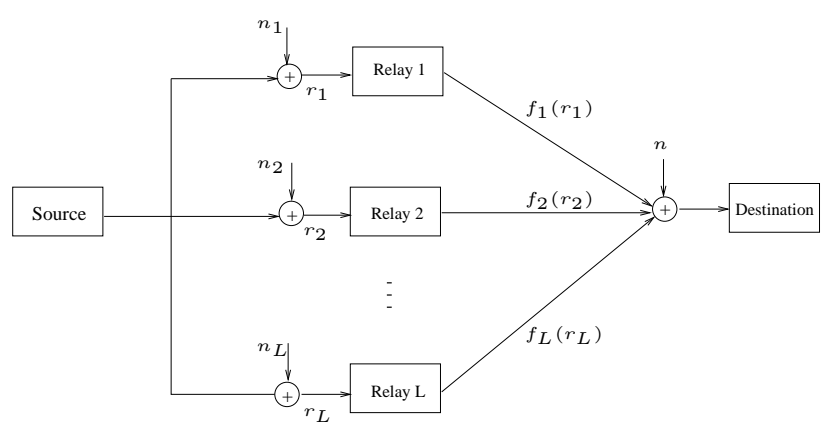

Fig. 5. Parallel Relay Network

of symbols from a Gaussian source is explained through the following. A Gaussian distribution is quantized into a number of states with the probability of the $i^{t h}$ state given by,

$$
\operatorname{Pr}\left(x_{i}\right)=\frac{1}{\sqrt{2 \pi P}} \int_{(i-1) \Delta x}^{i \Delta x} \exp \left(\frac{-x^{2}}{2 P}\right) d x .
$$

Suppose the source transmits symbols $x_{i}$ according to the probability distribution above, then the MAP detection rule at the relay is given by

$$
\widehat{X}(r)=\arg \max _{x_{i}} \operatorname{Pr}(x \mid r)
$$

In the limit $\Delta x \rightarrow 0, x$ and $r$ become jointly Gaussian. It is well known that the conditional mean $\mathcal{E}(x \mid r)$ maximizes the joint probability. Therefore $\mathcal{E}(x \mid r)$ which is also the MMSE estimate is the output of the ML detector. Thus for Gaussian inputs $A F, E F$ and $D F$ are identical, and the relay function is linear.

\section{Multiple Relay Network}

The design of relay functionalities assume more significance in networks with multiple relays as they offer enormous performance potential. Therefore it is important to develop insights into the optimal relay forwarding strategy for a general relay network. The network topology greatly determines the relay functionalities. For example, simple schemes like AF outperform decode and forward strategies in a parallel relay network [2]. However, decode and forward is the optimal strategy in a serial relay network. In this section we consider parallel and serial relay networks for uncoded source transmission. We also give an example of a hybrid network that consists of both parallel and serial subnetworks.

\section{A. Parallel Network}

A parallel relay network [7] is shown in Fig. 5. In the first slot, the relays observe $\left\{r_{i}\right\}_{i=1}^{L}$, the noisy version of the transmitted signal $x$.

$$
r_{i}=g_{i} x+n_{i}
$$

where $g_{i}$ is the gain of the link between the source and the $i^{t h}$ relay. $n_{i}$ denotes an additive white Gaussian noise with unit power. In the next slot, the destination receives the sum of all

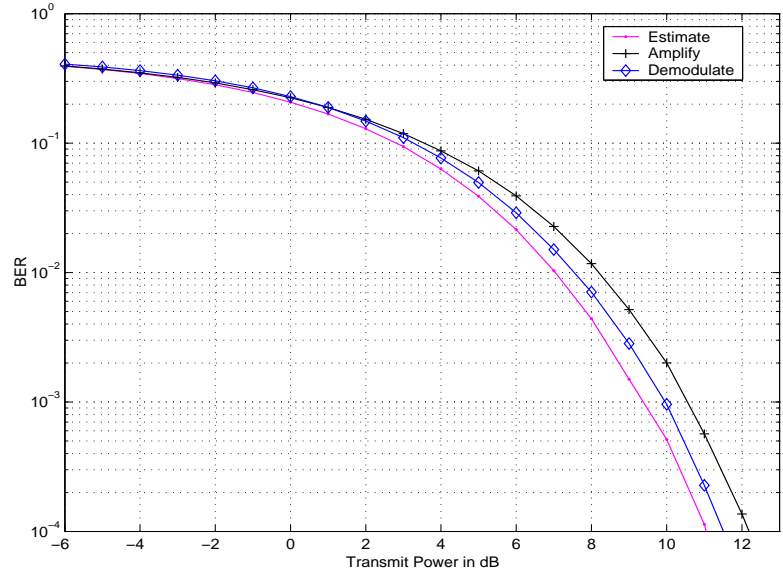

Fig. 6. BER of schemes in a parallel network $(L=2)$ for BPSK modulation.

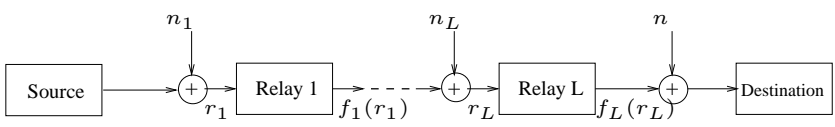

Fig. 7. Serial Relay Network

relay transmitted symbols along with its noise.

$$
y=\sum_{i=1}^{L} f_{i}\left(r_{i}\right)+n
$$

By viewing relay operation as an estimation we have,

$$
f_{i}\left(r_{i}\right)=f_{i}\left(g_{i} x+n_{i}\right)=\alpha_{i}\left(x+e_{i}\right),
$$

where $e_{i}$ is the uncorrelated estimation error at the $i^{t h}$ relay, $\alpha_{i}=\sqrt{\frac{P_{i}}{P+\mathrm{MSUE}_{i}}}$ and $\mathrm{MSUE}_{i}=\mathcal{E}\left[\left|e_{i}\right|^{2}\right]$, the mean square uncorrelated error associated with the $i^{t h}$ relay function. The received signal at the destination can be expressed as

$$
y=\sum_{i=1}^{L} \alpha_{i}\left(x+e_{i}\right)+n .
$$

From Theorem 1, we know that estimate and forward minimizes MSUE at the output of the relay. However, SNR optimality of EF cannot be guaranteed due to possible correlation between errors from different relays. Nevertheless, for most constellation schemes the error correlation is zero (for MPSK) or negligible (for MQAM) [5]. Fig. 6 displays the error probability for BPSK modulation for a parallel network with 2 relays for $P_{R}<P$. It can be seen that EF is the best performing scheme. Although soft information is important in a parallel network, we see that DF outperforms AF. This is because at low relay power, soft information gets degraded by the destination noise. However, if the power at the relays is high, AF outperforms DF. EF remains the best scheme for all scenarios.

\section{B. Serial Relay Network}

A serial relay network with Gaussian noise at all receivers in shown in Fig. 7. For unit channel gain for all the links, the 


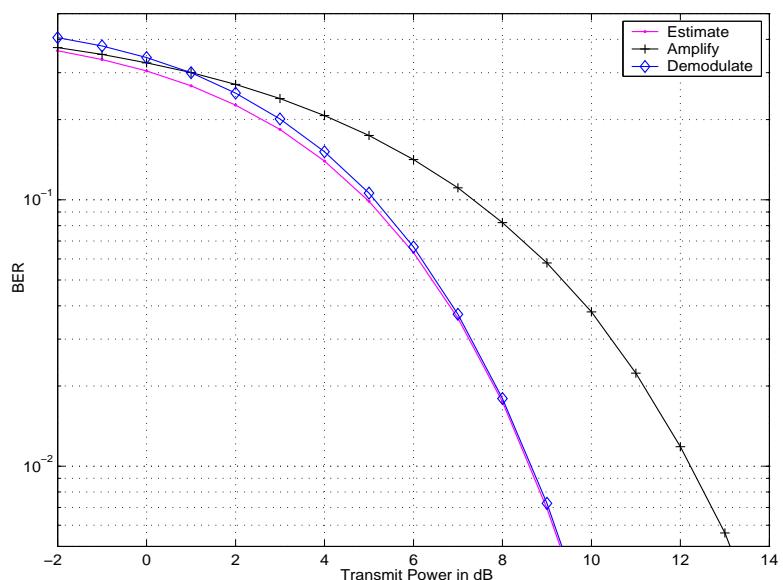

Fig. 8. BER of schemes in a serial network $(L=2)$ for BPSK modulation.

$\mathrm{AF}$ relay function for the $k^{t h}$ relay is

$$
f_{k}\left(r_{k}\right)=\beta r_{k},
$$

where $\beta^{2}=\frac{P}{P+1}$. The DF relay function for the $k^{t h}$ relay is given by

$$
f_{k}\left(r_{k}\right)=f_{k-1}\left(r_{k-1}\right)+d_{k}, \quad k=1 \ldots L,
$$

where $f_{0}\left(r_{0}\right)=x$ and $d_{k}$ represents the decision error at the $k^{t h}$ relay. The relay function for estimate and forward is

$$
f_{k}\left(r_{k}\right)=\alpha_{k} \mathcal{E}\left[x \mid r_{k}=f_{k-1}\left(r_{k-1}\right)+n_{k}\right],
$$

where $\alpha_{k}$ is such that $\mathcal{E}\left[\left|f_{k}\left(r_{k}\right)\right|^{2}\right]=P$ is satisfied.

Fig. 8 compares the error probability of the forwarding schemes for two serial relays. In a serial network, soft information is not greatly beneficial as there exists only one route. As expected, there is a huge performance degradation with $\mathrm{AF}$ due to its power inefficiency. At high $P$, DF performs almost identically as EF.

\section{Hybrid Relay Networks}

Although EF is the best scheme among the memoryless schemes for both parallel and serial relay networks, the performance gain over the best of $\mathrm{AF}$ and $\mathrm{DF}$ is limited. This is because for any scenario either DF or AF is near optimal. Now, consider a network consisting of both parallel and serial subnetworks as shown in Fig. 9. Due to the presence of parallel and serial elements together in the network, the relays need to provide soft information and also be power efficient. This is a scenario where EF obtains a large gain over the best of DF and AF. Fig. 10 compares the error performance of schemes for the hybrid network. It can be noticed that EF performs significantly better than the best of DF and AF. We expect the performance gain to increase for a large network with both parallel and serial elements.

\section{CONClusion}

In this paper, we addressed the relay functionality optimization problem from the perspective of soft information,

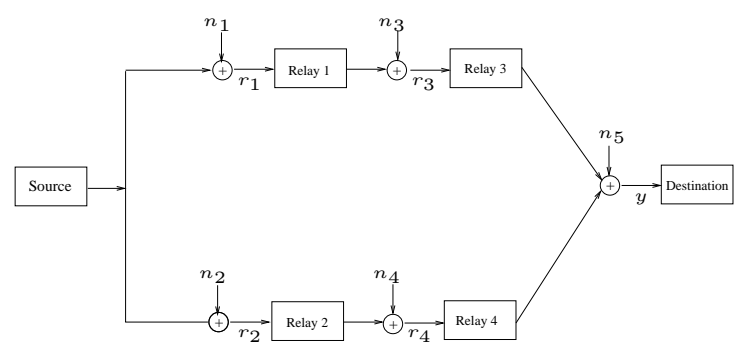

Fig. 9. Hybrid Relay Network

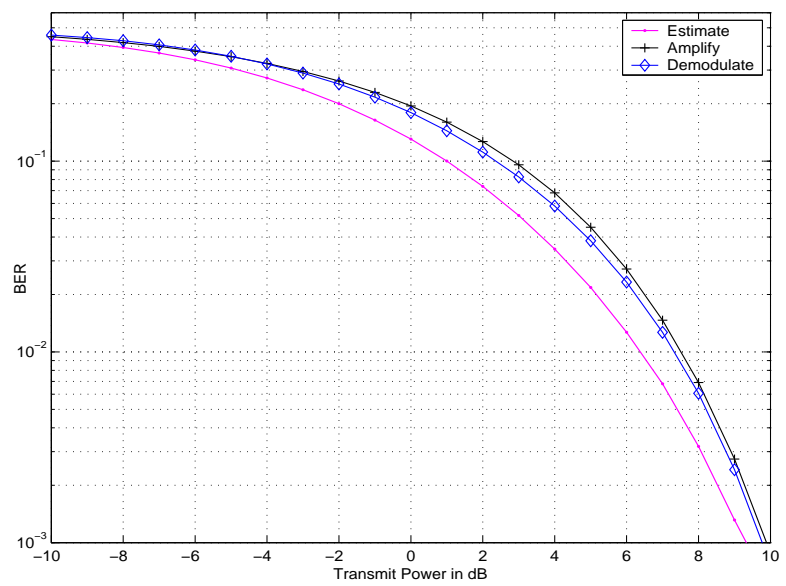

Fig. 10. BER of the forwarding schemes for BPSK modulation for the hybrid network in Fig. 9.

with SNR as a metric. We provided examples of existing forwarding strategies that provide hard and soft information. While soft information is always beneficial, it is expensive in terms of network resources. For a wireless network with a single relay and for an uncoded source, we determined that estimate and forward is SNR optimal. As it optimally trades off cost (power) for the degree of soft information, it is well suited to many network topologies like parallel and serial networks, and hybrid forms of these networks.

\section{REFERENCES}

[1] R. Ahlswede, N. Cai, S.Y.R. Li and R.W. Yeung, "Network Information Flow," IEEE Transactions on Information Theory, vol. 46, pp. 1204-1216, 2000.

[2] A.F. Dana, R. Gowaikar, B. Hassibi, M. Effros and M. Medard, "Should we break a wireless network into subnetworks?," in Allerton Conference on Communication, Control and Computing., 2003.

[3] D. Chen and J. N. Laneman, "Noncoherent Demodulation for Cooperative Diversity in Wireless Systems ," in Proceedings of Globecom, Dallas, Tx, Nov 2004.

[4] G. Kramer, M. Gastpar and P. Gupta, "Cooperative Strategies and Capacity Theorems for Relay Channels," IEEE Transactions on Information Theory, vol. 51, no. 9, pp. 3037-3063, Sep 2005.

[5] K. Gomadam and S. A. Jafar, "Optimal relay functionality for SNR maximization in memoryless relay networks," IEEE Journal on Selected Areas in Communications, vol. 25, no. 2, Feb 2007.

[6] I. Maric and R. Yates, "Forwarding Strategies for Gaussian Parallel-Relay Networks," in Proc. Conf. on Information Sciences and Systems (CISS), (Baltimore, MD), 2004.

[7] M. Gastpar and M. Vetterli, "On the Capacity of Large Gaussian Relay Networks ," IEEE Transactions on Information Theory, vol. 51, no.3, pp. 765-779, March 2005. 